\title{
Hematological Parameters and Prediabetes and Diabetes in Adults from the General Population: A Cross-Sectional Study
}

\author{
Meisinger $C^{1,2}$, Rückert IM ${ }^{1}$, Stöckl $D^{1}$, Thorand $B^{1,3}$, Peters $A^{1}$, Kowall $B^{4}$ and Rathmann $W^{4}$
}

${ }^{1}$ Helmholtz Zentrum München, German Research Center for Environmental Health (GmbH), Institute of Epidemiology II, Neuherberg, Germany

${ }^{2}$ Central Hospital of Augsburg, MONICA/KORA Myocardial Infarction Registry, Augsburg, Germany

${ }^{3}$ German Center for Diabetes Research (DZD e.V.), Neuherberg, Germany

${ }^{4}$ Institute of Biometrics and Epidemiology, German Diabetes Center, Leibniz Center for Diabetes Research at Heinrich Heine University, Düsseldorf, Germany

\section{Abstract}

Objective: We examined a possible association between hematological parameters and prediabetes (isolated impaired fasting glucose, isolated impaired glucose tolerance, and combined impaired fasting glucose/impaired glucose tolerance) as well as newly diagnosed and known diabetes in 32-81year old men and women from the general population.

Methods: This cross-sectional analysis was based on 2,963 participants of the Cooperative Health Research in the Region of Augsburg F4 Study conducted in 2006-2008 in Southern Germany. Hematological parameters were determined using impedance measurements.

Results: In multivariable multinomial regression analysis hemoglobin levels were significantly associated with isolated impaired glucose tolerance and combined impaired fasting glucose/impaired glucose tolerance compared to persons with normal glucose tolerance (Odds Ratio per standard deviation increment: 1.37 for isolated impaired glucose tolerance, and 1.63 for combined impaired fasting glucose/impaired glucose tolerance). Hematocrit values were significantly associated with isolated impaired glucose tolerance, combined impaired fasting glucose/impaired glucose tolerance, and newly diagnosed diabetes (Odds Ratio per standard deviation increment: 1.33 for isolated impaired glucose tolerance, 1.48 for combined impaired fasting glucose/impaired glucose tolerance, and 1.29 for newly diagnosed diabetes). White blood cell count showed a significant association with isolated impaired glucose tolerance (Odds Ratio per standard deviation increment 1.46; 95\% confidence interval 1.27-1.66), newly diagnosed diabetes (Odds Ratio 1.55; 95\% confidence interval 1.31-1.83), and known diabetes (Odds Ratio 1.43; 95\% confidence interval 1.23-1.66). While we found no association between platelet count and prediabetes or diabetes, mean platelet volume was significantly related to known diabetes (Odds Ratio per standard deviation increment 1.19; $95 \%$ confidence interval 1.02-1.38). Most associations were more pronounced in women than men.

Conclusion: Clinically relevant hematological parameters may play a role in the early development of type 2 diabetes. Further research is necessary to study the gender-specific contribution of hematological parameters to the pathogenesis of prediabetic states and finally to the manifestation of type 2 diabetes.

Keywords: Hematological parameters; Population-based study; Prediabetes; Diabetes; Risk factor; Epidemiology; Gender

\section{Background}

The prevalence of type 2 diabetes is increasing worldwide and exhibits a challenge on the health care system as well as on the public health and socioeconomic development of all nations [1]. The causes of type 2 diabetes are multifactorial and result from a combination of environmental and genetic risk factors [1]. The implementation of preventive measures in populations and the identification of high risk groups, which mostly benefit from such activities, will be the key point for the early prevention of type 2 diabetes and its complications. Within the last years, a number of potential risk factors for type 2 diabetes have been identified. In this context, one's attention was particularly turned on lifestyle risk factors, inflammatory parameters, metabolic abnormalities, and genetic risk factors, many of which have been found to be independently associated with type 2 diabetes [2]. Among those risk factors, routinely measured hematological parameters, such as White Blood Cell (WBC) count and hematocrit (HCT) level were associated with insulin resistance and incident type 2 diabetes [3-8]. Hematocrit is positively correlated with hyperinsulinemia and risk factors associated with insulin resistance, e.g. high blood pressure, elevated serum triglycerides, low HDL cholesterol, and central obesity and could therefore be related to insulin resistance. Furthermore, hematocrit is a major determinant of blood viscosity. Increased blood viscosity also contributes to the development of insulin resistance [38]. In addition, chronic inflammation is involved in the pathogenesis of type 2 diabetes and evidence from epidemiological studies suggests an association between total WBC or leukocyte count, a non-specific marker of inflammation, and diabetes risk [2]. However, only a few prior studies investigated, whether selected hematological parameters such as WBC count, Mean Platelet Volume (MPV) or red cell count are related to prediabetic states [9-12].

*Corresponding author: Meisinger C, Central Hospital of Augsburg, MONICA/KORA Myocardial Infarction Registry, Stenglinstr. 2, D-86156 Augsburg, Germany, Tel: 0049/821/400-4373; Fax:0049/821/400-2838 E-mail: christa.meisinger@helmholtz-muenchen.de

Received January 09, 2014; Accepted February 08, 2014; Published February 13,2014

Citation: Meisinger C, Rückert IM, StöckI D, Thorand B, Peters A, et al. (2014) Hematological Parameters and Prediabetes and Diabetes in Adults from the General Population: A Cross-Sectional Study. J Diabetes Metab 5: 335 doi:10.4172/2155-6156.1000335

Copyright: (c) 2014 Meisinger C, et al. This is an open-access article distributed under the terms of the Creative Commons Attribution License, which permits unrestricted use, distribution, and reproduction in any medium, provided the original author and source are credited. 
Citation: Meisinger C, Rückert IM, StöckI D, Thorand B, Peters A, et al. (2014) Hematological Parameters and Prediabetes and Diabetes in Adults from the General Population: A Cross-Sectional Study. J Diabetes Metab 5: 335. doi:10.4172/2155-6156.1000335

So far, to the best of our knowledge, there is no population-based study, which investigated the association between various routinely measured hematological parameters and prediabetes. Therefore, the aim of the present study was to investigate the associations of hematological parameters, including HCT, Hemoglobin (HGB), Platelet Count (PC), Mean Platelet Volume (MPV), and WBC count with prediabetic groups (Isolated Impaired Fasting Glucose (i-IFG), Isolated Impaired Glucose Tolerance (i-IGT), and combined IFG/IGT) and newly diagnosed as well as known diabetes mellitus in a population of 32-81year old men and women in Southern Germany. To investigate whether there are sex-specific particularities, in addition all analyses were performed separately for men and women.

\section{Research Design and Methods}

Data are based on the Cooperative Health Research in the Region of Augsburg (KORA) F4 study (2006-2008), a follow-up of the KORA S4 study, a population-based health survey conducted in 1999-2001. Of all 4,261 participants of the S4 baseline study, 3,080 also participated in the 7-year follow-up F4 study (response 79.6\%) [13]. Persons were considered ineligible for F4 if they lived outside the study region or were completely lost to follow-up $(n=206,5 \%)$, or had demanded deletion of their address data $(n=12,0.2 \%)$. Furthermore, 176 persons (4\%) had died during follow-up time. Of the remaining 3,867 eligible persons, 174 could not be contacted, 218 were unable to come to the study center because they were too ill or had no time, and 395 were not willing to participate in this follow-up. The current study was restricted to 2,963 participants aged 32-81 (1534 women and 1429 men) without missing values on any of the analytical variables $(n=117)$. The investigations were carried out in accordance with the Declaration of Helsinki, including written informed consent of all participants. All study methods were approved by the Ethics committee of the Bavarian Chamber of Physicians, Munich.

After an overnight fast of at least 10 hours, all nondiabetic participants underwent a standard 75-g Oral Glucose Tolerance Test (OGTT) [14]. In persons with physician diagnosed known diabetes no OGTT was carried out. Newly Diagnosed Diabetes (NDD; $\geq 7.0 \mathrm{mmol} / \mathrm{l}$ fasting plasma glucose or $\geq 11.1 \mathrm{mmol} / \mathrm{l} 2$-h post glucose load), i-IFG (fasting plasma glucose $\geq 6.1 \mathrm{mmol} / \mathrm{l}$, but $<7.0 \mathrm{mmol} / \mathrm{l}$ and 2-h post glucose load $<7.8 \mathrm{mmol} / \mathrm{l}$ ), i-IGT (fasting plasma glucose $<6.1 \mathrm{mmol} / \mathrm{l}$ and 2-h post glucose load $\geq 7.8 \mathrm{mmol} / \mathrm{l}$, but $<11.1 \mathrm{mmol} / \mathrm{l}$ ), IFG/IGT, and normal glucose tolerance (NGT; fasting plasma glucose $<6.1$ $\mathrm{mmol} / \mathrm{l}$ and 2 - $\mathrm{h}$ post glucose load $<7.8 \mathrm{mmol} / \mathrm{l}$ ) were defined according to the 1999 WHO diagnostic criteria as described elsewhere [13].

Information on sociodemographic variables, lifestyle, and other risk factors was gathered during a standardized interview. All participants underwent an extensive standardized medical examination as described in more detail elsewhere [15]. Hypertension was defined as blood pressure values $\geq 140 / 90 \mathrm{mmHg}$ and/or use of antihypertensive medication, given that the individuals were aware of being hypertensive. Individuals who participated in leisure time physical training during summer and winter and were active for at least one hour per week in either season were classified as being physically active. A regular smoker was defined as a participant who smoked at least one cigarette per day. Alcohol intake was categorized into three categories: men: $0,0.1-39.9$ or $\geq 40 \mathrm{~g}$ /day; women: $0,0.1-19.9$ or $\geq 20$ g/day.

\section{Clinical chemical measurements}

A fasting venous blood sample was obtained from all study participants while sitting. All parameters were measured immediately. Blood glucose was analyzed using a hexokinase method (GLU Flex, Dade Behring). Total serum cholesterol, and HDL cholesterol analyses were carried out using a CHOD-PAP method (Dade Behring). Triacylglycerol was measured with the GPO-PAP-method (TGL Flex, Dade Behring). Serum creatinine was determined using a modified kinetic Jaffé reaction [13]. HbAlc was measured with a reverse-phase cation-exchange High Pressure Liquid Chromotography (HPLC) method (Menarini, analyzer HA 8160). Hematological parameters were determined from fresh venous EDTA blood samples by impedance measurements (Coulter ${ }^{\circledR}$ LH 780, Beckman Coulter GmbH, Krefeld) [16].

\section{Statistical analyses}

The $\mathrm{Chi}^{2}$-Test was used to test differences in prevalence. A general linear model was used to compare means (F-Test). We performed multinomial logistic regression analyses using the SAS procedure PROC LOGISTIC to study the association of different hematological parameters with different categories of impaired glucose regulation [17]. In these analyses the categorical dependent outcome had six groups (NGT, i-IFG, i-IGT, IFG/IGT, NDD, and known diabetes) and the reference group for comparison was NGT. Two models were fitted: The first model included the respective hematological parameter, age (continuous) and sex. The second, multivariable multinomial regression model included in addition to the respective hematological parameter age, sex, hypertension (yes/no), physical activity (active/inactive), regular smoking (yes/no), alcohol intake (gender-specific categories), total cholesterol (continuous), HDL cholesterol (continuous), serum creatinine (continuous), and BMI (continuous). Odds Ratios (OR) and 95\% Confidence Intervals (95\% CI) were computed for a 1 Standard Deviation (SD) increase of the respective hematological parameter. An OR is a measure of association between an exposure and an outcome. The OR represents the odds that an outcome (in this study i-IFG, i-IGT, IFG/IGT, NDD, and known diabetes) will occur given a particular exposure (in this study an increase of the hematological parameters HCT, HGB, PC, MPV, and WBC count), compared to the odds of the outcome occurring in the absence of that exposure. The OR is calculated by the formula:

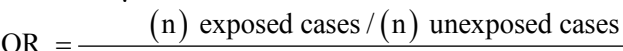

(n) exposed non - cases / (n) unexposed non - cases

Linearity was checked by including a quadratic term of the respective hematological variable in the model. Because the p-values of the quadratic terms were not significant, linearity could be assumed for all investigated parameters. We tested for interaction between the different hematological parameters and sex on the risk of impaired glucose regulation categories. A p-value of $<0.05$ was considered statistically significant. All analyses were performed with SAS software version 9.2. (SAS Institute, Inc., Cary, NC, USA).

\section{Results}

Characteristics of the study sample according to glucose tolerance categories are shown in Table 1. Persons with prediabetes (i-IFG, i-IGT, or IFG/IGT), and newly diagnosed diabetes, but not persons with known diabetes had higher HCT, and HGB levels than reference subjects with NGT. As compared to the NGT group, persons with prediabetic states, NDD or known diabetes had lower platelet counts and higher WBC counts, they were older, more likely to be less educated, had a higher BMI or waist circumference, as well as a higher systolic and diastolic blood pressure. Individuals with prediabetes, NDD or known diabetes 
Citation: Meisinger C, Rückert IM, StöckI D, Thorand B, Peters A, et al. (2014) Hematological Parameters and Prediabetes and Diabetes in Adults from the General Population: A Cross-Sectional Study. J Diabetes Metab 5: 335. doi:10.4172/2155-6156.1000335

Page 3 of 6

\begin{tabular}{|c|c|c|c|c|c|c|c|}
\hline & $\begin{array}{l}\text { NGT } \\
(n=2,130)\end{array}$ & $\begin{array}{l}i-I F G \\
(n=113)\end{array}$ & $\begin{array}{l}\text { i-IGT } \\
(n=315)\end{array}$ & $\begin{array}{l}\text { IFG/IGT } \\
(n=69)\end{array}$ & $\begin{array}{l}\text { NDD } \\
(n=108)\end{array}$ & $\begin{array}{l}\text { Known T2DM } \\
(n=228)\end{array}$ & $P$ value \\
\hline Age (years) & $52.8(12.6)$ & $61.3(10.8)$ & $63.7(11.3)$ & $64.3(9.1)$ & $65.3(10.4)$ & $67.5(9.1)$ & $<0.0001$ \\
\hline Male sex (\%) & 45.5 & 66.4 & 46.7 & 60.9 & 56.5 & 59.2 & $<0.0001$ \\
\hline Education (<12 years, \%) & 56.1 & 55.8 & 66.7 & 59.4 & 63.0 & 72.4 & $<0.0001$ \\
\hline BMI $\left(\mathrm{kg} / \mathrm{m}^{2}\right)$ & $26.6(4.3)$ & $29.8(4.6)$ & $29.5(4.8)$ & $30.8(5.0)$ & $31.1(4.4)$ & $31.2(5.4)$ & $<0.0001$ \\
\hline Waist circumference $(\mathrm{cm})$ & $90.5(12.8)$ & $102.4(12.5)$ & $98.7(14.2)$ & $104.9(13.4)$ & $104.5(11.2)$ & $105.2(12.7)$ & $<0.0001$ \\
\hline Systolic blood pressure $(\mathrm{mm} \mathrm{Hg})$ & $119.1(17.4)$ & $130.8(19.4)$ & $126.4(19.3)$ & $134.2(16.6)$ & $131.6(17.6)$ & $132.8(19.9)$ & $<0.0001$ \\
\hline Diastolic blood pressure $(\mathrm{mm} \mathrm{Hg})$ & $74.7(9.7)$ & $77.4(9.9)$ & $75.5(10.6)$ & $81.0(9.3)$ & $77.1(11.1)$ & $74.5(10.5)$ & $<0.0001$ \\
\hline Actual hypertension (\%) & 27.5 & 54.0 & 54.9 & 69.6 & 77.8 & 79.4 & $<0.0001$ \\
\hline Hematocrit (I/l) & $0.41(0.03)$ & $0.42(0.03)$ & $0.42(0.03)$ & $0.43(0.03)$ & $0.42(0.04)$ & $0.41(0.04)$ & $<0.0001$ \\
\hline Hemoglobin $(\mathrm{g} / \mathrm{l})$ & $139.9(12.6)$ & $143.6(11.1)$ & $142.5(12.0)$ & $146.3(11.2)$ & $143.6(13.0)$ & $139.4(12.8)$ & $<0.0001$ \\
\hline Platelet count (/nl) & $257.1(61.2)$ & $254.0(61.8)$ & $251.9(68.4)$ & $234.6(55.7)$ & $249.1(63.4)$ & $237.4(62.4)$ & $<0.0001$ \\
\hline Mean platelet volume (fl) & $8.9(0.9)$ & $8.9(1.1)$ & $8.9(0.9)$ & $9.1(0.9)$ & $8.9(1.0)$ & $9.1(1.0)$ & 0.0310 \\
\hline White blood cell count (/nl) & $5.6(1.3)$ & $5.8(1.3)$ & $6.0(1.3)$ & $6.0(1.3)$ & $6.6(1.3)$ & $6.4(1.3)$ & $<0.0001$ \\
\hline Total cholesterol (mmol/l) & $5.6(1.0)$ & $5.8(1.1)$ & $5.8(1.1)$ & $5.9(1.0)$ & $5.7(1.1)$ & $5.2(1.0)$ & $<0.0001$ \\
\hline HDL cholesterol (mmol/l) & $1.5(0.4)$ & $1.3(0.3)$ & $1.4(0.4)$ & $1.3(0.3)$ & $1.2(0.3)$ & $1.3(0.3)$ & $<0.0001$ \\
\hline Triacylglycerol $(\mathrm{mmol} / \mathrm{l})^{\mathrm{a}}$ & $1.1(1.7)$ & $1.6(1.6)$ & $1.4(1.6)$ & $1.8(1.8)$ & $1.8(1.8)$ & $1.6(1.8)$ & $<0.0001$ \\
\hline Creatinine $(\mu \mathrm{mol} / /)^{a}$ & $76.9(1.2)$ & $81.1(1.2)$ & $79.4(1.2)$ & $83.5(1.2)$ & $83.6(1.3)$ & $86.7(1.3)$ & $<0.0001$ \\
\hline Fasting glucose $(\mathrm{mmol} / \mathrm{l})^{\mathrm{a}}$ & $5.1(1.1)$ & $6.3(1.0)$ & $5.3(1.1)$ & $6.5(1.0)$ & $6.7(1.2)$ & $7.5(1.3)$ & $<0.0001$ \\
\hline 2h-glucose $\left(\mathrm{mmol} / \mathrm{l}^{\mathrm{a}}\right)$ & $5.3(1.3)$ & $6.0(1.2)$ & $8.9(1.1)$ & $9.2(1.1)$ & $11.6(1.3)$ & -- & $<0.0001$ \\
\hline $\mathrm{HbA1c}$ value (\%) & $5.4(0.3)$ & $5.7(0.3)$ & $5.6(0.3)$ & $5.9(0.3)$ & $6.2(0.9)$ & $6.8(1.1)$ & $<0.0001$ \\
\hline $\mathrm{HbA} 1 \mathrm{c}$ value $(\mathrm{mmol} / \mathrm{mol})$ & $35.1(3.3)$ & $38.8(3.5)$ & $37.5(3.5)$ & $40.7(3.8)$ & $44.2(9.6)$ & $51.1(11.8)$ & $<0.0001$ \\
\hline \multicolumn{8}{|l|}{ Alcohol intake (\%) } \\
\hline 0 g/day & 28.8 & 31.0 & 31.1 & 24.6 & 30.6 & 42.1 & 0.0025 \\
\hline $0.1-19.9 \mathrm{~g}(\mathrm{w}) /$ day/0.1-39.9g/day (m) & 54.0 & 45.1 & 53.0 & 50.7 & 47.2 & 43.0 & \\
\hline$\geq 20.0 \mathrm{~g} /$ day $(\mathrm{w}) / \geq 40 \mathrm{~g} /$ day $(\mathrm{m})$ & 17.2 & 23.9 & 15.9 & 24.6 & 22.2 & 14.9 & \\
\hline Regular smoking (\%) & 17.7 & 9.7 & 6.7 & 8.7 & 11.1 & 10.5 & $<0.0001$ \\
\hline Physically active during leisure time (\%) & 58.3 & 45.1 & 49.5 & 53.6 & 47.2 & 36.4 & $<0.0001$ \\
\hline
\end{tabular}

NGT: Normal Glucose Tolerance; I-IFG: Isolated Impaired Fasting Glucose; I-IGT: Isolated Impaired Glucose Tolerance; NDD: Newly Detected Diabetes; ageometric mean (geometric SD)

Table 1: Study characteristics - means (SD) or percentages - by NGT, i-IFG, i-IGT, IFG/IGT, NDD, and known Type 2 Diabetes Mellitus (T2DM), persons aged 32-81 years $(\mathrm{N}=2,963)$, KORA F4 study.

more often suffered from hypertension. Prediabetic states and NDD as well as known diabetes were related to lower HDL cholesterol, higher triacylglycerols, higher creatinine values, non-smoking, and lack of physical activity. As compared to the NGT group, persons with prediabetes or NDD had higher total cholesterol values, while persons with known diabetes had lower total cholesterol values.

There were significant interactions between HCT, HGB, and WBC count and sex regarding the risk of some prediabetic/diabetic states. Therefore, the analysis was also stratified by sex.

The results of the multinomial regression analysis for the total sample are given in Table 2 . In the age- and sex-adjusted model the OR per SD increment of HGB was 1.50 (95\% CI 1.28-1.75) for i-IGT, 1.92 (95\% CI 1.41-2.62) for IFG/IGT, and 1.48 (95\% CI 1.15-1.90) for NDD. Further adjustment for smoking status, alcohol intake, physical activity, actual hypertension, total cholesterol, HDL cholesterol, creatinine values, and BMI somewhat attenuated the associations, but the ORs per SD HGB increment were still significantly increased for the i-IGT and IFG/IGT group compared to persons with NGT, while the association with NDD became non-significant. In stratified analysis (Table 3) these associations were found in women only; in men no significant association between HGB and prediabetic states and NDD could be found after multivariable adjustment. However, in males there was a significantly inverse association between HGB levels and known diabetes even after multivariable adjustment (Table 3).
In the total sample HCT values were significantly associated with i-IGT, IFG/IGT, and NDD in the age- and sex-adjusted analysis (Table 2). After multivariable adjustment, the associations remained significant for all three groups: the OR per SD increment of HCT was 1.33 (95\% CI 1.12-1.57) for i-IGT, 1.48 (95\% CI 1.08-2.04) for IFG/ IGT, and 1.29 (95\% CI 1.00-1.66) for NDD. The same significant associations were also found in women (Table 3). Contrary, in men no associations between HCT values and prediabetic states and NDD were found, but there was a significant inverse relationship of HCT with known diabetes in the age- and sex-adjusted and multivariable adjusted models (Table 3 ).

There was no significant association between platelet count and prediabetes groups as well as NDD in the total sample; however, in the age- and sex-adjusted analysis PC was significantly inversely associated with known diabetes (OR 0.84; 95\% CI 0.72-0.99) (Table 2). This association lost significance after multivariable adjustment in the total sample. In stratified analysis there was no relationship of PC with prediabetes, NDD and known diabetes in women, but in men, we found no association between PC and prediabetes as well as NDD, but a significantly inverse association between PC and known diabetes even after multivariable adjustment (Table 3).

MPV showed a significant association only with known diabetes in the age- and sex-adjusted model and in the multivariable adjusted model (OR per SD increment 1.19; 95\% 1.02-1.38) in the total sample (Table 2). In stratified analyses, there was also a significant association with known diabetes in both sexes in the age-adjusted models. After 
Citation: Meisinger C, Rückert IM, StöckI D, Thorand B, Peters A, et al. (2014) Hematological Parameters and Prediabetes and Diabetes in Adults from the General Population: A Cross-Sectional Study. J Diabetes Metab 5: 335. doi:10.4172/2155-6156.1000335

Page 4 of 6

\begin{tabular}{|c|c|c|c|c|c|c|}
\hline & $\begin{array}{l}\text { NGT } \\
(n=2,130)\end{array}$ & $\begin{array}{l}\text { i-IFG } \\
(n=113)\end{array}$ & $\begin{array}{l}i-I G T \\
(n=315)\end{array}$ & $\begin{array}{l}\text { IFG/IGT } \\
(n=69)\end{array}$ & $\begin{array}{l}\text { NDD } \\
(n=108)\end{array}$ & $\begin{array}{l}\text { Known diabetes } \\
(\mathrm{n}=228)\end{array}$ \\
\hline Hemoglobin & OR $(95 \% \mathrm{Cl})$ & OR $(95 \% \mathrm{Cl})$ & OR $(95 \% \mathrm{Cl})$ & OR $(95 \% \mathrm{Cl})$ & OR $(95 \% \mathrm{Cl})$ & OR $(95 \% \mathrm{Cl})$ \\
\hline aModel 1 & 1.00 & $1.19(0.93-1.51)$ & $1.50(1.28-1.75)$ & $1.92(1.41-2.62)$ & $1.48(1.15-1.90)$ & $0.92(0.77-1.09)$ \\
\hline${ }^{b}$ Model 2 & 1.00 & $1.03(0.80-1.33)$ & $1.37(1.16-1.62)$ & $1.63(1.18-2.26)$ & $1.26(0.97-1.63)$ & $0.90(0.75-1.09)$ \\
\hline \multicolumn{7}{|l|}{ Hematocrit } \\
\hline aModel 1 & 1.00 & $1.15(0.91-1.46)$ & $1.43(1.23-1.67)$ & $1.69(1.25-2.29)$ & $1.47(1.15-1.87)$ & $0.91(0.77-1.07)$ \\
\hline bModel 2 & 1.00 & $1.02(0.79-1.30)$ & $1.33(1.12-1.57)$ & $1.48(1.08-2.04)$ & $1.29(1.00-1.66)$ & $0.89(0.74-1.07)$ \\
\hline \multicolumn{7}{|l|}{ Platelet count } \\
\hline aModel 1 & 1.00 & $1.11(0.92-1.33)$ & $0.99(0.87-1.12)$ & $0.78(0.60-1.03)$ & $1.00(0.82-1.22)$ & $0.84(0.72-0.99)$ \\
\hline${ }^{b}$ Model 2 & 1.00 & $1.11(0.92-1.35)$ & $0.99(0.86-1.12)$ & $0.80(0.60-1.06)$ & $1.02(0.84-1.25)$ & $0.88(0.75-1.03)$ \\
\hline \multicolumn{7}{|c|}{ Mean platelet volume } \\
\hline aModel 1 & 1.00 & $1.10(0.91-1.33)$ & $1.04(0.86-1.27)$ & $1.24(0.99-1.56)$ & $1.04(0.86-1.27)$ & $1.27(1.11-1.46)$ \\
\hline${ }^{b}$ Model 2 & 1.00 & $1.08(0.89-1.31)$ & $1.02(0.90-1.16)$ & $1.18(0.93-1.49)$ & $0.98(0.80-1.20)$ & $1.19(1.02-1.38)$ \\
\hline \multicolumn{7}{|c|}{ White blood cell count } \\
\hline aModel 1 & 1.00 & $1.15(0.93-1.44)$ & $1.47(1.30-1.67)$ & $1.37(1.06-1.76)$ & $1.68(1.44-1.96)$ & $1.61(1.40-1.84)$ \\
\hline bModel 2 & 1.00 & $1.09(0.85-1.38)$ & $1.46(1.27-1.66)$ & $1.27(0.97-1.68)$ & $1.55(1.31-1.83)$ & $1.43(1.23-1.66)$ \\
\hline
\end{tabular}

NGT: Normal Glucose Tolerance; i-IFG: Isolated Impaired Fasting Glucose; i-IGT: Isolated Impaired Glucose Tolerance; NDD: Newly Detected Diabetes;

Odds ratio (OR) expressed per SD-increment; significant findings are highlighted in bold characters

aModel 1: adjusted for age and sex

'Model 2: adjusted for age, sex, actual hypertension, regular smoking, physical activity, alcohol intake, total cholesterol, HDL cholesterol, creatinine, and BMI

Table 2: Association of hematological parameters with i-IFG, i-IGT, IFG/IGT, NDD, and known diabetes (comparisons with NGT), KORA F4 study, total sample (N=2,963).

\begin{tabular}{|c|c|c|c|c|c|c|}
\hline & NGT & i-IFG & i-IGT & IFG/IGT & NDD & Known diabetes \\
\hline Men $(n=1,429)$ & $(n=969)$ & $(n=75)$ & $(n=147)$ & $(n=42)$ & $(n=61)$ & $(n=135)$ \\
\hline Hemoglobin & OR $(95 \% \mathrm{Cl})$ & OR $(95 \% \mathrm{Cl})$ & OR $(95 \% \mathrm{Cl})$ & OR $(95 \% \mathrm{Cl})$ & OR $(95 \% \mathrm{Cl})$ & OR $(95 \% \mathrm{Cl})$ \\
\hline aModel 1 & 1.00 & $1.09(0.81-1.46)$ & $1.39(1.11-1.74)$ & $1.54(1.04-2.27)$ & $1.37(0.99-1.89)$ & $0.80(0.64-0.99)$ \\
\hline${ }^{\mathrm{b}}$ Model 2 & 1.00 & $0.96(0.70-1.31)$ & $1.23(0.97-1.57)$ & $1.31(0.86-1.97)$ & $1.17(0.83-1.64)$ & $0.78(0.62-0.98)$ \\
\hline \multicolumn{7}{|l|}{ Hematocrit } \\
\hline a Model 1 & 1.00 & $1.06(0.80-1.42)$ & $1.30(1.05-1.62)$ & $1.35(0.92-1.96)$ & $1.35(0.98-1.85)$ & $0.77(0.62-0.96)$ \\
\hline${ }^{\mathrm{b}}$ Model 2 & 1.00 & $0.95(0.69-1.29)$ & $1.16(0.92-1.47)$ & $1.16(0.77-1.73)$ & $1.18(0.84-1.64)$ & $0.75(0.59-0.94)$ \\
\hline \multicolumn{7}{|l|}{ Platelet count } \\
\hline aModel 1 & 1.00 & $1.06(0.84-1.33)$ & $1.02(0.86-1.22)$ & $0.76(0.53-1.08)$ & $0.91(0.70-1.20)$ & $0.76(0.62-0.95)$ \\
\hline${ }^{\mathrm{b}}$ Model 2 & 1.00 & $1.10(0.87-1.39)$ & $1.04(0.86-1.25)$ & $0.79(0.55-1.14)$ & $0.97(0.74-1.28)$ & $0.79(0.64-0.98)$ \\
\hline \multicolumn{7}{|c|}{ Mean platelet volume } \\
\hline${ }^{a}$ Model 1 & 1.00 & $1.09(0.86-1.38)$ & $0.98(0.82-1.18)$ & $1.27(0.95-1.71)$ & $1.00(0.76-1.30)$ & $1.27(1.05-1.53)$ \\
\hline${ }^{\mathrm{b}}$ Model 2 & 1.00 & $1.05(0.83-1.35)$ & $0.95(0.78-1.15)$ & $1.18(0.86-1.60)$ & $0.90(0.68-1.19)$ & $1.19(0.97-1.45)$ \\
\hline \multicolumn{7}{|c|}{ White blood cell count } \\
\hline${ }^{a}$ Model 1 & 1.00 & $1.03(0.78-1.35)$ & $1.37(1.16-1.61)$ & $1.18(0.85-1.63)$ & $1.41(1.16-1.71)$ & 1.34 (1.13-1.59) \\
\hline${ }^{\mathrm{b}}$ Model 2 & 1.00 & $1.01(0.74-1.36)$ & $1.32(1.11-1.58)$ & $1.11(0.77-1.59)$ & $1.33(1.07-1.66)$ & $1.18(0.98-1.43)$ \\
\hline Women $(n=1,534)$ & $(n=1,161)$ & $(n=38)$ & $(n=168)$ & $(n=27)$ & $(n=47)$ & $(n=93)$ \\
\hline Hemoglobin & OR $(95 \% \mathrm{Cl})$ & OR $(95 \% \mathrm{Cl})$ & OR $(95 \% \mathrm{Cl})$ & OR $(95 \% \mathrm{Cl})$ & OR $(95 \% \mathrm{Cl})$ & OR $(95 \% \mathrm{Cl})$ \\
\hline${ }^{a}$ Model 1 & 1.00 & $1.33(0.86-2.07)$ & $1.64(1.30-2.07)$ & $2.72(1.61-4.62)$ & $1.66(1.11-2.47)$ & $1.19(0.89-1.58)$ \\
\hline${ }^{\mathrm{b}}$ Model 2 & 1.00 & $1.16(0.73-1.82)$ & $1.60(1.25-2.06)$ & $2.44(1.41-4.23)$ & $1.50(0.98-2.29)$ & $1.18(0.86-1.62)$ \\
\hline \multicolumn{7}{|l|}{ Hematocrit } \\
\hline${ }^{\text {a }}$ Model 1 & 1.00 & $1.26(0.83-1.93)$ & $1.60(1.28-2.00)$ & $2.41(1.44-4.01)$ & $1.63(1.11-2.41)$ & $1.20(0.90-1.59)$ \\
\hline${ }^{\mathrm{b}}$ Model 2 & 1.00 & $1.11(0.71-1.72)$ & $1.57(1.23-2.00)$ & $2.20(1.29-3.76)$ & $1.56(1.02-2.38)$ & $1.21(0.88-1.66)$ \\
\hline \multicolumn{7}{|l|}{ Platelet count } \\
\hline${ }^{a}$ Model 1 & 1.00 & $1.19(0.87-1.63)$ & $0.96(0.80-1.14)$ & $0.81(0.53-1.25)$ & $1.12(0.84-1.50)$ & $0.96(0.76-1.21)$ \\
\hline${ }^{\mathrm{b}}$ Model 2 & 1.00 & $1.16(0.84-1.60)$ & $0.95(0.79-1.14)$ & $0.81(0.52-1.27)$ & $1.13(0.83-1.53)$ & $1.01(0.78-1.29)$ \\
\hline \multicolumn{7}{|c|}{ Mean platelet volume } \\
\hline${ }^{a}$ Model 1 & 1.00 & $1.12(0.82-1.54)$ & $1.10(0.93-1.29)$ & $1.19(0.82-1.72)$ & $1.10(0.82-1.47)$ & $1.26(1.02-1.55)$ \\
\hline${ }^{\mathrm{b}}$ Model 2 & 1.00 & $1.11(0.81-1.52)$ & $1.09(0.92-1.29)$ & $1.16(0.79-1.70)$ & $1.06(0.79-1.44)$ & $1.18(0.94-1.48)$ \\
\hline \multicolumn{7}{|c|}{ White blood cell count } \\
\hline a Model 1 & 1.00 & $1.37(0.95-1.98)$ & $1.51(1.25-1.82)$ & $1.70(1.13-2.55)$ & $2.19(1.68-2.84)$ & $2.07(1.67-2.57)$ \\
\hline${ }^{\mathrm{b}}$ Model 2 & 1.00 & $1.16(0.76-1.76)$ & $1.56(1.27-1.91)$ & $1.54(0.98-2.41)$ & $1.87(1.39-2.52)$ & $1.84(1.43-2.38)$ \\
\hline
\end{tabular}

NGT: Normal Glucose Tolerance; i-IFG: Isolated Impaired Fasting Glucose; i-IGT: Isolated Impaired Glucose Tolerance; NDD: Newly Detected Diabetes; Odds ratio (OR) expressed per SD-increment; significant findings are highlighted in bold characters; aModel 1: adjusted for age ; ${ }^{\mathrm{b}}$ Model 2: adjusted for age, actual hypertension, regular smoking, physical activity, alcohol intake, total cholesterol, HDL cholesterol, creatinine, and BMI

Table 3: Gender-specific association of hematological parameters with i-IFG, i-IGT, IFG/IGT, NDD, and known diabetes (comparisons with NGT), KORA F4 study.

multivariable adjustment this relationship became non-significant in both men and women (Table 3).

In the whole sample WBC count was significantly associated with
i-IGT, IFG/IGT, NDD, and known diabetes in the age- and sex-adjusted analysis. After multivariable adjustment the associations with i-IGT (OR 1.46; 95\% CI 1.27-1.66), NDD (OR 1.55; 95\% CI 1.31-1.83), and 
known diabetes (OR 1.43; 95\% CI 1.23-1.66) remained significant, but the relationship with IFG/IGT lost significance (Table 2). In stratified multivariable analysis, there was a significant association between WBC count and i-IGT and NDD in men, while in women a significant association between WBC count and i-IGT, NDD, and known diabetes was found (Table 3).

\section{Discussion}

In the present population based study, HGB and HCT concentrations were significantly associated with i-IGT and IFG/ IGT after multivariable adjustment. WBC count showed a significant relationship with i-IGT, NDD, and known diabetes. In addition, while we observed no significant association between platelet count and impaired glucose regulation, MPV was significantly associated with known diabetes only. The observed associations were stronger in women than in men.

Most studies on this issue included men only or did not conduct sex-specific analysis [5-12]. Thus, the present study confirms prior findings on an association between HCT, WBC count and diabetes and extends the current knowledge regarding the sex-specific relationship of different hematological parameters and prediabetic states in persons from the general population.

Several prior studies investigated the association between HCT levels and the incidence of type 2 diabetes [5-8]. In the Atherosclerosis Risk in Communities (ARIC) study elevated levels of HCT and whole blood viscosity were associated with insulin resistance and were independent predictors of type 2 diabetes [8]. A study conducted in Pima Indians has found, that HCT predicted diabetes after multivariable adjustment (HRR per $5 \%$ difference in blood hematocrit 1.40; 95\% CI 1.28-1.53) [7]. This was also the case, when restricting the analysis to persons with HCT measurements within the normal range [7]. In the present study, HCT levels were associated with NDD only and not with known diabetes. This could possibly be due to the fact that in our study persons with known and thus treated diabetes had lower HCT values than all other glucose tolerance groups and even those with NGT. Furthermore, we could show a significant association between HCT levels and i-IGT and IFG/IGT even after adjustment for a variety of confounding variables.

We also found a significant relationship of HGB concentrations and i-IGT and IFG/IGT. So far, no population-based studies on the association between HGB values and diabetes or prediabetic states are available. A very recent prospective study conducted in Japanese community-dwelling persons reported that hematological parameters including red blood cell count, HCT, and HGB are independently associated with insulin resistance [18]. Furthermore two other studies showed a relationship between HGB and metabolic syndrome $[19,20]$.

Prior studies have found that hematological parameters play an important role in insulin resistance [21,22]. The pathophysiological mechanisms by which HCT could influence glucose metabolism are not entirely clear. Insulin increases the transcapillary movement of albumin and as a consequence the plasma volume will decrease and cause an increase of the HCT [23]. Higher HCT levels imply higher blood viscosity, which may predispose to insulin resistance and type 2 diabetes by limiting delivery of glucose, insulin, and oxygen to metabolically active tissues [24]. HGB is an important Nitric Oxide (NO) buffer and a modulator of NO bioavailability and thus involved in the regulation of endothelial function [25]. Additionally, there is a positive association between hemoglobin and sCD40L level, a proinflammatory marker released by activated platelets, which predicts cardiovascular events in patients with high-risk atherosclerotic lesions and is elevated in persons with prediabetes and diabetes [26-28].

In the present study we could show that WBC count was significantly associated with NDD, known diabetes, and i-IGT. This finding is in accordance with prior studies, suggesting an association between WBC count and diabetes as well as IGT [9,10,29-31]. WBC count is a marker of systemic inflammation and may thus be involved in the pathophysiology of prediabetic states and subsequently in the manifestation of diabetes $[29,30]$.

While we found no association between PC and impaired glucose regulation, MPV was associated with known diabetes after multivariable adjustment in the present study. Several earlier studies have reported a positive association between MPV and fasting plasma glucose levels in persons with diabetes [32,33]. Coban et al. found in their study, that subjects with IGT tend to have increased MPV levels [11]. MPV is an indicator for an increased platelet activity and thus thrombogenic activation, which may play a role in the development of vascular complications in persons with type 2 diabetes. A recent study found that MPV was significantly higher in persons with diabetes than in non-diabetic persons and that MPV was strongly correlated to fasting and postprandial glucose levels as well as HbAlc values [34]. In another study conducted in 1876 Japanese subjects MPV was higher in persons with prediabetes in comparison with NGT subjects [35]. Our results underscore the findings of prior studies indicating that elevated MPV may be involved in the development of vascular complications in persons with known diabetes.

In the present study, the associations between the hematological parameters and prediabetic/diabetic states were stronger in women than in men. Hence, hematological parameters may play a possible role in the early development of type 2 diabetes, particularly in women. Furthermore, there were strong associations found between the single hematological parameters and i-IGT, but no relationships with i-IFG. Although the prediabetc states i-IGT and i-IFG are associated with a high risk for type 2 diabetes, they manifest distinct metabolic abnormalities [36]. Both insulin resistance and impaired beta cell function contribute to the development of IGT and IFG, but subjects with IGT have predominantly increased insulin resistance in skeletal muscle, while persons with IFG have predominantly increased hepatic insulin resistance [36]. Further research is necessary to confirm or refute the present findings and to investigate the role of hematological parameters in the etiology of peripheral insulin resistance, particularly in women.

The cross-sectional design of the study represents a limitation, implicating that cause and effect relationships cannot be discerned. We cannot exclude that unknown risk factors may have biased or confounded the present analysis. Because the analyses were based on a follow-up examination of the population-based KORA S4 study, it could be argued whether or not the responders are representative of the initial population-based sample. The non-responders in the KORA F4 study were for example younger, had lower total cholesterol values, were more often smokers, and physically inactive than the participants. But with respect to other important risk factors for diabetes such as BMI and hypertension there were no differences between the two groups [37]. The strength of the study is the inclusion of a large number of individuals randomly drawn from the general population, and the availability of data on lifestyle and multiple metabolic risk factors. 
Citation: Meisinger C, Rückert IM, StöckI D, Thorand B, Peters A, et al. (2014) Hematological Parameters and Prediabetes and Diabetes in Adults from the General Population: A Cross-Sectional Study. J Diabetes Metab 5: 335. doi:10.4172/2155-6156.1000335

\section{Conclusions}

The hematological parameters HGB and HCT were significantly associated with IGT, particularly in women from the general population. WBC count was related to IGT as well as NDD and known diabetes also more pronounced in women. Thus, it seems that hematological parameters may play a role in the early development of type 2 diabetes. Further research is necessary to study the sex-specific contribution of hematological parameters to the pathogenesis of prediabetic states and finally to the manifestation of type 2 diabetes.

\section{Acknowledgments}

The KORA research platform is financed by the Helmholtz Zentrum München, German Research Center for Environmental Health $(\mathrm{GmbH})$, which is funded by the German Federal Ministry of Education, Science, Research and Technology and by the State of Bavaria. The Diabetes Study was funded by a German Diabetes Foundation project grant (201/05/07). This study was supported in part by a gran from the German Federal Ministry of Education and Research (BMBF) to the German Center for Diabetes Research (DZD e.V.). We thank all members of the Helmholtz Zentrum München and the field staff in Augsburg who were involved in the conduct of the study.

\section{References}

1. Hu FB (2011) Globalization of diabetes: the role of diet, lifestyle, and genes. Diabetes Care 34: 1249-1257.

2. Bi Y, Wang T, Xu M, Xu Y, Li M, et al. (2012) Advanced research on risk factors of type 2 diabetes. Diabetes Metab Res Rev 28: 32-39.

3. Medalie JH, Papier CM, Goldbourt U, Herman JB (1975) Major factors in the development of diabetes mellitus in 10,000 men. Arch Intern Med 135: 811 817.

4. Wilson PW, McGee DL, Kannel WB (1981) Obesity, very low density lipoproteins, and glucose intolerance over fourteen years: The Framingham Study. Am J Epidemiol 114: 697-704.

5. Wannamethee SG, Perry IJ, Shaper AG (1996) Hematocrit and risk of NIDDM Diabetes 45: 576-579.

6. Nakanishi N, Suzuki K, Tatara K (2004) Haematocrit and risk of developmen of Type 2 diabetes mellitus in middle-aged Japanese men. Diabet Med 21: 476-482.

7. Tulloch-Reid MK, Hanson RL, Saremi A, Looker HC, Williams DE, et al. (2004) Hematocrit and the incidence of type 2 diabetes in the pima indians. Diabetes Care 27: 2245-2246.

8. Tamariz LJ, Young JH, Pankow JS, Yeh HC, Schmidt MI, et al. (2008) Blood viscosity and hematocrit as risk factors for type 2 diabetes mellitus: the atherosclerosis risk in communities (ARIC) study. Am J Epidemiol 168: 11531160.

9. Gokulakrishnan K, Deepa R, Sampathkumar R, Balasubramanyam M, Mohan $V$ (2009) Association of leukocyte count with varying degrees of glucose intolerance in Asian Indians: the Chennai Urban Rural Epidemiology Study (CURES-26). Metab Syndr Relat Disord 7: 205-210.

10. Nakanishi N, Yoshida H, Matsuo Y, Suzuki K, Tatara K (2002) White blood-cell count and the risk of impaired fasting glucose or Type II diabetes in middleaged Japanese men. Diabetologia 45: 42-48

11. Coban E, Kucuktag S, Basyigit S (2007) Platelet activation in subjects with impaired glucose tolerance. Platelets 18: 591-594.

12. Simmons D (2010) Increased red cell count in diabetes and pre-diabetes. Diabetes Res Clin Pract 90: e50-53.

13. Rathmann W, Kowall B, Tamayo T, Giani G, Holle R, et al. (2012) Hemoglobin $\mathrm{A} 1 \mathrm{c}$ and glucose criteria identify different subjects as having type 2 diabetes in middle-aged and older populations: the KORA S4/F4 Study. Ann Med 44: 170-177.

14. Rathmann W, Haastert B, Icks A, Löwel H, Meisinger C, et al. (2003) High prevalence of undiagnosed diabetes mellitus in Southern Germany: target populations for efficient screening. The KORA survey 2000. Diabetologia 46 : 182-189.

15. Meisinger C, Thorand B, Schneider A, Stieber J, Döring A, et al. (2002) Sex differences in risk factors for incident type 2 diabetes mellitus: the MONICA Augsburg cohort study. Arch Intern Med 162: 82-89.

16. Tan BT, Nava AJ, George TI (2011) Evaluation of the Beckman Coulter UniCe DxH 800, Beckman Coulter LH 780, and Abbott Diagnostics Cell-Dyn Sapphire hematology analyzers on adult specimens in a tertiary care hospital. Am J Clin Pathol 135: 939-951.

17. Hosmer DW Jr, Lemeshow S, Sturdivant RX (2013) Applied Logistic Regression ( $3^{\text {rd }}$ edn), (C) John Wiley \& Sons, Inc., New York.

18. Kawamoto R, Tabara Y, Kohara K, Miki T, Kusunoki T, et al. (2013) Hematological parameters are associated with metabolic syndrome in Japanese community-dwelling persons. Endocrine 43: 334-341.

19. Lohsoonthorn V, Jiamjarasrungsi W, Williams MA (2007) Association of Hematological Parameters with Clustered Components of Metabolic Syndrome among Professional and Office Workers in Bangkok, Thailand. Diabetes Metab Syndr 1: 143-149.

20. Lin JD, Chiou WK, Chang HY, Liu FH, Weng HF, et al (2006) Association of hematological factors with components of the metabolic syndrome in older and younger adults. Aging Clin Exp Res 18: 477-484.

21. Wang YY, Lin SY, Liu PH, Cheung BM, Lai WA (2004) Association between hematological parameters and metabolic syndrome components in a Chinese population. J Diabetes Complications 18: 322-327.

22. Choi KM, Lee J, Kim YH, Kim KB, Kim DL, et al. (2003) Relation between insulin resistance and hematological parameters in elderly Koreans-Southwest Seoul (SWS) Study. Diabetes Res Clin Pract 60: 205-212.

23. Hilsted J, Christensen NJ (1992) Dual effect of insulin on plasma volume and transcapillary albumin transport. Diabetologia 35: 99-103.

24. Stuart J, Kenny MW (1980) Blood rheology. J Clin Pathol 33: 417-429.

25. Jia L, Bonaventura C, Bonaventura J, Stamler JS (1996) S-nitrosohaemoglobin: a dynamic activity of blood involved in vascular control. Nature 380: 221-226.

26. Bautista LE, Atwood JE, O'Malley PG, Taylor AJ (2004) Association between C-reactive protein and hypertension in healthy middle-aged men and women. Coron Artery Dis 15: 331-336.

27. Tasci I, Dogru T, Sonmez A, Genc H, Kilic S, et al. (2006) Soluble CD40 ligand levels in otherwise healthy subjects with impaired fasting glucose. Mediators Inflamm 2006: 32508.

28. Gokulakrishnan K, Deepa R, Mohan V, Gross MD (2006) Soluble P-selectin and CD40L levels in subjects with prediabetes, diabetes mellitus, and metabolic syndrome--the Chennai Urban Rural Epidemiology Study. Metabolism 55: 237 242.

29. Ford ES (2002) Leukocyte count, erythrocyte sedimentation rate, and diabetes incidence in a national sample of US adults. Am J Epidemiol 155: 57-64.

30. Du X, Zhu B, Hu G, Mao W, Wang S, et al. (2009) U-shape association between white blood cell count and the risk of diabetes in young Chinese adults. Diabet Med 26: 955-960.

31. Twig G, Afek A, Shamiss A, Derazne E, Tzur D, et al. (2013) White blood cells count and incidence of type 2 diabetes in young men. Diabetes Care 36 : 276-282.

32. Papanas N, Symeonidis G, Maltezos E, Mavridis G, Karavageli E, et al. (2004) Mean platelet volume in patients with type 2 diabetes mellitus. Platelets 15 475-478.

33. Shah B, Sha D, Xie D, Mohler ER 3rd, Berger JS (2012) The relationship between diabetes, metabolic syndrome, and platelet activity as measured by mean platelet volume: the National Health And Nutrition Examination Survey, 1999-2004 Diabetes Care 35: 1074-1078.

34. Kodiatte TA, Manikyam UK, Rao SB, Jagadish TM, Reddy M, et al. (2012) Mean platelet volume in Type 2 diabetes mellitus. J Lab Physicians 4: 5-9.

35. Shimodaira M, Niwa T, Nakajima K, Kobayashi M, Hanyu N, et al. (2013) Correlation between mean platelet volume and fasting plasma glucose levels in prediabetic and normoglycemic individuals. Cardiovascular Diabetology 12: 14.

36. Abdul-Ghani MA, DeFronzo RA (2009) Pathophysiology of prediabetes. Curr Diab Rep 9: 193-199.

37. Meisinger C, Strassburger K, Heier M, Thorand B, Baumeister SE, et al. (2010) Prevalence of undiagnosed diabetes and impaired glucose regulation in 35-59-year-old individuals in Southern Germany: the KORA F4 Study. Diabet Med 27: 360-362. 